\title{
A New Dissimilarity Measure for Trajectories with Applications in Anomaly Detection
}

\author{
Dustin L. Espinosa-Isidrón and Edel B. García-Reyes \\ Advanced Technologies Application Center (CENATAV), 7a \# 21812 e/ 218 y 222, \\ Rpto. Siboney, Playa, C.P. 12200, La Habana, Cuba \\ \{despinosa, edel\}@cenatav.co.cu
}

\begin{abstract}
Trajectory clustering has been used to very effectively in the detection of anomalous behavior in video sequences. A key point in trajectory clustering is how to measure the (dis)similarity between two trajectories. This paper deals with a new dissimilarity measure for trajectory clustering, giving the same importance to differences and similarities between the trajectories. Experimental results in the task of anomalous detection via hierarchical clustering shows the validity of the proposed approach.
\end{abstract}

Keywords: Trajectory Clustering, Dissimilarity, Anomaly Detection.

\section{Introduction}

Video surveillance is a research field that has received much interest over the last years. Parking lot surveillance, traffic monitoring, and crime prevention are among the applications of video surveillance systems. A key task that could help improve the effectiveness of these systems is the automatic detection of anomalous behaviors. Trajectory clustering has been established as an effective tool to address the task. A fundamental issue in trajectory clustering is how to measure the (dis)similarity between the trajectories.

In this work we propose a new dissimilarity measure for trajectories, namely Dissimilarity for Trajectories(DT). The core of DT is a non-symmetric dissimilarity which yields the same importance to differences and similarities between the trajectories. All measures are tested in the task of anomaly detection via trajectory clustering. The selected data sets contain different amounts of normal trajectories with different amounts of abnormal trajectories.

The remainder of this paper is organized as follows. Sec. 2 describes the representation and dissimilarity for trajectories, including the proposed dissimilarity measure. Anomaly detection via trajectory clustering is presented alongside experimental results in Sec. 3. Finally, Sec. 4. concludes the paper.

\section{2 (Dis)Similarity Measures for Trajectories}

Trajectory Representation: Usually a trajectory is represented as a sequence $S=\left(s_{1}, s_{2}, \ldots, s_{h}\right)$, where each $s_{i}, 1 \leq i \leq h$, is a point in a multidimensional space containing information about the moving object at time $i$. Most

I. Bloch and R.M. Cesar, Jr. (Eds.): CIARP 2010, LNCS 6419, pp. 193 201, 2010.

(C) Springer-Verlag Berlin Heidelberg 2010 


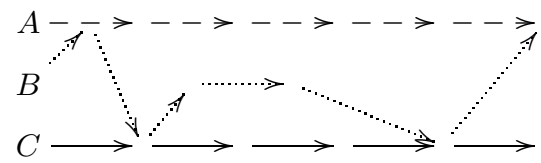

Fig. 1. Three trajectories $(A, B$ and $C)$, near in space, but trajectory $B$ has a very different directional behavior

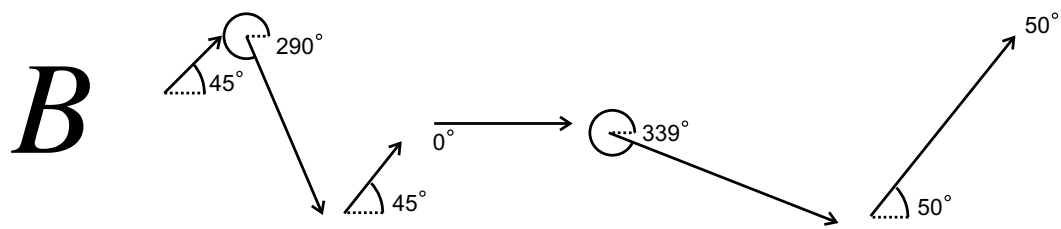

Fig. 2. Calculation of the direction in each point of the trajectory B. In each point $b_{i}$, the direction is represented as the angle clockwise with the right horizontal of $\left(x_{b i}, y_{b i}\right)$. To subtract two directions $d_{b i}, d_{a j}$ we take the minimum between $\left|d_{b i}-d_{a j}\right|$ and $\left|360-d_{b i}-d_{a j}\right|$

of the research done until now for trajectory analysis, is only based on the $\operatorname{position}\left(s_{i}=\left(x_{s i}, y_{s i}\right)\right)$ at time $i[1][2$. Note that time information is used implicitly, since in general, trajectories are sampled at equal time rates. Other features one should consider are the direction and the velocity of the objects. Obtaining both features depends on the tracker and not all tracking algorithms records this information. The significance of using direction comes from the fact that two trajectories can be very close in the space domain, but have a very different directional behavior (see figure 1). The direction at time $i$ can be estimated using the position at time $i+1$, as the angle clockwise with the right horizontal of $\left(x_{s i}, y_{s i}\right)$ as shown in figure 2. For the case of the velocity you also need to know the sampling rate. In this work we represent $s_{i}$ as a point in a 4 dimensional space $s_{i}=\left(x_{s i}, y_{s i}, t_{s i}, d_{s i}\right)$, encoding information about the $\operatorname{position}\left(x_{s i}, y_{s i}\right)$, time $\left(t_{s i}\right)$ and direction $\left(d_{s i}\right)$ of the moving object. In addition, each feature is normalized dividing by its standard deviation.

Previous Work: The Euclidian dissimilarity for trajectories, the Longest Common SubSequence(LCSS) and the Dynamic Time Warping(DTW) are the three most widely studied (dis)similarity measure for trajectories in the literature [1] [4. This section introduces these widely used (dis)similarity measures and discusses their main features.

Euclidean(EU) Dissimilarity is defined as the sum of the distances between corresponding points: $E u(S, T)=\sum_{i=1}^{h} D\left(s_{i}, t_{i}\right)$, where $h$ is the length of the trajectories and $D$ is the Euclidean distance between $s_{i}$ and $t_{i}[\underline{3}$. Note that 
this measure assumes that both trajectories have the same length. This is not a common scenario due to speed variations and occlusions[5]. Another known problem of the Euclidean Measure is that it cannot handle local time shifting [1]. However it is simple to implement and very fast $O(h)$.

Longest Common Subsequence(LCSS) Similarity is a variation of the Edit Distance on strings [3]. Let $S=\left(s_{1}, s_{2}, \ldots, s_{h}\right)$ and $T\left(t_{1}, t_{2}, \ldots, t_{l}\right)$ two trajectories, $\delta$ and $\epsilon$ two user-defined parameters, then LCSS is defined as:

$$
\operatorname{Lcss}(S, T)= \begin{cases}0 & \text { if }|S|=0 \text { or }|T|=0 \\ 1+\operatorname{Lcss}(\operatorname{Rest}(S), \operatorname{Rest}(T)) & \text { if }\left|t_{s 1}-t_{t 1}\right| \leq \delta \text { and } \\ & \left|x_{s 1}-x_{t 1}\right| \leq \epsilon \text { and }\left|y_{s 1}-y_{t 1}\right| \leq \epsilon \\ \max (\operatorname{Lcss}(\operatorname{Rest}(S), T), & \text { otherwise } \\ \operatorname{Lcss}(S, \operatorname{Rest}(T))) & \end{cases}
$$

where $\operatorname{Rest}(S)=\left(s_{2}, \ldots, s_{h}\right)$.

The main idea of LCSS is to allow the trajectories to stretch and to allow some points to remain unmatched [1]. The value of $\operatorname{Lcss}(S, T)$ is the length of the longest matching subsequence of $S$ and $T$ as indicated by its name. This measure has proven great effectiveness in the presence of noisy points [3]. The main drawback of $\operatorname{Lcss}(S, T)$ is that it does not penalize unmatched subsequences, given no information of how separated the unmatched subsequences are. Work in [3] tries to solve this, but they use a fixed penalty, no matter the difference of the subsequences. In addition, $\operatorname{Lcss}(S, T)$ in its original form, doesn't take the direction into account, and may fail to separate two trajectories near in space but very different directional behavior (see figure 1). Also, it should be noticed that the user needs to define $\epsilon$ and $\delta$ parameters. Last, the time complexity of $\operatorname{Lcss}(S, T)$ is $O(|S|+|T|)$ applying dynamic programming techniques and with a small $\delta[1]$.

Dynamic Time Warping(DTW) Dissimilarity as LCSS allows warping in time, but contrary to LCSS doesn't allow points to remain unmatched[1]. DTW is defined as:

$$
\operatorname{Dtw}(S, T)= \begin{cases}0 & \text { if }|S|=0 \text { and }|T|=0 \\ \infty & \text { if }|S|=0 \text { or }|T|=0 \\ D\left(s_{1}, t_{1}\right)+\min (\operatorname{Dtw}(\operatorname{Rest}(S), \operatorname{Rest}(T)), & \text { otherwise } \\ \operatorname{Dtw}(\operatorname{Rest}(S), T), \operatorname{Dtw}(S, \operatorname{Rest}(T))) & \end{cases}
$$

The main idea of DTW is to duplicate some points to handle local time shifting. The general criticism to DTW in the literature is its sensitivity to noisy points, since all points need to be matched 3] 1. In addition, we point out that DTW penalize long trajectories. Two very similar long trajectories may have similar DTW value that two dissimilar but much smaller trajectories. As all of above measures if information about the direction is not included in the trajectories representation, DTW may fail to separate two trajectories near in space but very different directional behavior. The time complexity of $\operatorname{Dtw}(S, T)$ is $O(|S||T|)$ applying dynamic programming as with LCSS. 


\subsection{Proposed Approach}

With the purpose of solving the above mentioned problems, we developed a new dissimilarity measure for trajectories. This measure should be able to handle trajectories of different lengths. Moreover, it shouldn't be influenced by the length of the given trajectories, in the sense that a pair of long (dis)similar trajectories should have an analogous dissimilarity value as another pair of smaller ones. Besides, similar subsequences should be rewarded, and dissimilar ones penalized.

Given two trajectories $S$ and $T$, and a boolean user-defined parameter $m$, the Dissimilarity for Trajectories(DT) is defined as:

$$
D T(S, T, m)= \begin{cases}\min (A D T(S, T) /|S|, A D T(T, S) /|T|) & \text { if } m=\text { true } \\ \max (A D T(S, T) /|S|, A D T(T, S) /|T|) & \text { if } m=\text { false }\end{cases}
$$

where:

$$
A D T(S, T)= \begin{cases}N \text { earest }\left(s_{1}, T\right) & \text { if }|S|=1 \\ \sum_{i=1}^{|S|} D\left(s_{i}, t_{1}\right) & \text { if }|T|=1 \\ \min \left(D\left(s_{1}, t_{1}\right)+A D T(\operatorname{Rest}(S), T),\right. & \text { otherwise } \\ \quad A D T(S, \operatorname{Rest}(T)) & \end{cases}
$$

and Nearest $(s, T)=\min _{t \in T} D(s, t)$

Nearest $(s, T)$ returns the distance of the nearest point to $s$ in the sequence $T$. The function $A D T(S, T)$ (Asymmetric Dissimilarity for Trajectories) is a nonsymmetric dissimilarity measure that quantifies how far $S$ is from $T$. This is done by finding the subsequences of $T$ (allowing replication of points in $T$ as in DTW, as well as allowing some points of $T$ remain unmatched as in LCSS) passing nearest to $S$, and summing the Euclidean distances of corresponding points (see figures 3 and (4). If $S$ is a subsequent of $T$, then $A D T(S, T)$ is 0 . On the other hand, $D T(S, T)$ normalizes the result of $A D T(S, T)$ and $A D T(T, S)$ dividing by the size of $S$ and $T$ respectively. Furthermore, let the user to chose what is more relevant, the differences $(\max )^{1}$ or the similarities(min) among the trajectories. The time complexity of $D T(S, T)$ is $O(|S||T|)$ applying similar dynamic programming techniques as with DTW and LCSS.

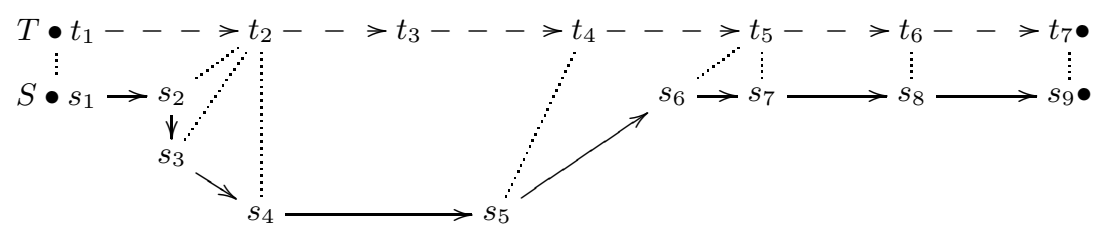

Fig. 3. Resultant matching points in $A D T(S, T)$. The dissimilarity value is equivalent to $E u\left(S, T^{\prime}\right)$, where $T^{\prime}=\left(t_{1}, t_{2}, t_{2}, t_{2}, t_{4}, t_{5}, t_{5}, t_{6}, t_{7}\right)$. The points $t_{2}$ and $t_{5}$ were duplicated and $t_{3}$ was left unmatched.

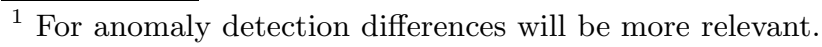




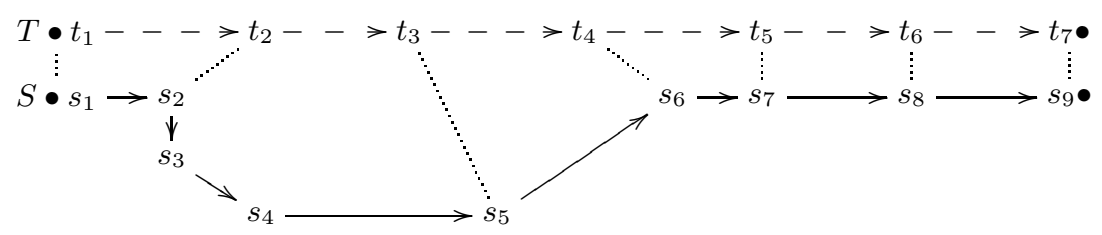

Fig. 4. Resultant matching points in $A D T(T, S)$. The dissimilarity value is equivalent to $E u\left(S^{\prime}, T\right)$, where $S^{\prime}=\left(s_{1}, s_{2}, s_{5}, s_{6}, s_{7}, s_{8}, s_{9}\right)$. The points $t_{3}$ and $t_{4}$ were left unmatched.

To sum up briefly, the new measure is able to work with trajectories of different sizes(contrary to EU), it is not influenced by the size of the trajectories, since the value is normalized(contrary to DTW, LCSS), and allows the user to specify the relevance of differences and similarities. Besides, only the less dissimilar(most similar) subsequences are used in the computation of the dissimilarity(contrary to EU, DTW), and Euclidian measure is used to penalize the differences(contrary to $\mathrm{LCSS})$.

\section{Experimental Results in Anomaly Detection}

The automatic anomaly detection task implies finding objects moving with an unusual(abnormal, infrequent) pattern. Trajectory clustering provides a practical approach for the detection of this unusual(abnormal) motion patterns in video sequences. The main idea is that larger groups are considered as normal trajectories while singletons or very small groups are considered as abnormal trajectories. In this section a trajectory clustering algorithm is selected for the task of anomaly detection. The (dis)similarity measures presented above are tested with a selected clustering algorithm, in a previously used data set, and experimental results are shown.

Single-Link $(S L)[6$ is a well known and widely used hierarchical clustering algorithm. Given a set of objects $O$, a dissimilarity measure $d$, and a stopping criteria $\theta$, the $S L$ algorithm is defined as:

1. Represent each object of $O$ as a singleton cluster.

2. Select the two objects with minimum $d$ that are in different clusters.

3. Join the corresponding clusters.

4. Stop if $\theta$ is true, else go to step 2 .

This algorithm has several desirables properties that make it a practical choice for anomaly detection. First, it has a very easy implementation. Moreover, it can discover clusters of any shape and size. Furthermore, it has a very easy interpretation: objects separated from the rest(i.e. anomalies) are joined last, this 
Table 1. Results using EU. For each entry (rows = anomalies; columns = groups of normal trajectories) ten runs were performed and the results averaged.

\begin{tabular}{|c|c|c|c|c|c|c|c|c|c|c|}
\hline \multicolumn{11}{|c|}{ FALSE POSITIVES. The total average over 100 cases: $\mathbf{6 . 0 6} \%$} \\
\hline Anomalies & \multicolumn{10}{|c|}{ Groups of normal trajectories } \\
\hline & 1 & 2 & 3 & 4 & 5 & 6 & 7 & 8 & 9 & 10 \\
\hline 1 & 0.30 & 0.00 & 0.00 & 0.00 & 0.00 & 0.00 & 0.00 & 0.00 & 0.00 & 0.00 \\
\hline 2 & 0.50 & 6.80 & 4.20 & 1.65 & 7.35 & 6.45 & 4.40 & 8.35 & 6.25 & 6.95 \\
\hline$\overline{3}$ & 9.00 & 2.90 & 14.03 & 7.23 & 6.90 & 3.57 & 2.30 & 5.07 & 1.53 & 3.57 \\
\hline 4 & 8.70 & 5.43 & 4.83 & 8.25 & 6.65 & 5.78 & 5.83 & 6.35 & 11.20 & 5.05 \\
\hline 5 & 7.96 & 2.90 & 10.08 & 6.16 & 7.56 & 5.48 & 10.20 & 7.66 & 3.76 & 3.54 \\
\hline 6 & 9.38 & 5.32 & 8.22 & 6.87 & 5.25 & 5.93 & 8.43 & 6.67 & 3.33 & 9.90 \\
\hline 7 & 5.14 & 7.37 & 11.27 & 8.53 & 6.00 & 8.40 & 6.89 & 7.30 & 9.46 & 5.03 \\
\hline$\overline{8}$ & 6.85 & 8.46 & 6.68 & 10.01 & 8.53 & 11.08 & 7.04 & 6.53 & 7.94 & 5.74 \\
\hline 9 & 7.49 & 4.79 & 5.78 & 7.86 & 7.70 & 4.27 & 6.73 & 6.07 & 8.80 & 7.84 \\
\hline 10 & 11.34 & 4.92 & 7.00 & 6.39 & 10.10 & 3.95 & 8.26 & 8.48 & 5.91 & 6.51 \\
\hline \multicolumn{11}{|c|}{ TRUE POSITIVES. The total averag } \\
\hline Anomalies & \multicolumn{10}{|c|}{ Groups of normal trajectories } \\
\hline 1 & 90.00 & 100.00 & 100.00 & 100.00 & 100.00 & 100.00 & 100.00 & 98.75 & 100.00 & $\overline{100.00}$ \\
\hline$\overline{2}$ & 100.00 & 100.00 & 96.67 & 100.00 & 100.00 & 98.33 & 100.00 & 98.75 & 93.33 & 99.00 \\
\hline 3 & 100.00 & 100.00 & 96.67 & 95.00 & 96.00 & 98.33 & 98.57 & 98.75 & 97.78 & 99.00 \\
\hline$\overline{4}$ & 100.00 & 90.00 & 100.00 & 100.00 & 100.00 & 100.00 & 100.00 & 98.75 & 96.67 & 97.00 \\
\hline 5 & 80.00 & 100.00 & 96.67 & \begin{tabular}{|l|}
92.50 \\
\end{tabular} & 98.00 & \begin{tabular}{|l|}
98.33 \\
\end{tabular} & 95.71 & 98.75 & \begin{tabular}{|l|}
96.67 \\
\end{tabular} & 95.00 \\
\hline 6 & 90.00 & 100.00 & 100.00 & 95.00 & 94.00 & 95.00 & 97.14 & 92.50 & 97.78 & 98.00 \\
\hline 7 & 100.00 & 95.00 & 100.00 & 97.50 & 96.00 & 90.00 & 95.71 & 97.50 & 96.67 & 97.00 \\
\hline 8 & 80.00 & 100.00 & 96.67 & 95.00 & 100.00 & 95.00 & 95.71 & 97.50 & 97.78 & 98.00 \\
\hline 9 & 100.00 & 100.00 & 96.67 & 100.00 & 88.00 & 98.33 & 88.57 & 95.00 & 97.78 & 97.00 \\
\hline 10 & 100.00 & \begin{tabular}{|l|}
90.00 \\
\end{tabular} & 93.33 & 95.00 & 92.00 & 90.00 & 92.86 & 95.00 & \begin{tabular}{|l|}
98.89 \\
\end{tabular} & 96.00 \\
\hline
\end{tabular}

is very important since minimizes the risk of false positives. The main drawback is that it suffers from the chaining effect, but if the clusters are well separated(the dissimilarity measure should be able to separate the anomalies from the rest), the algorithm performs well [6].

Experimental Results: A synthetic data set, first introduced in 2], was selected to test the effectiveness of the (dis)similarity measures presented in this work, for the task of anomaly detection. It contains "100 different experimental cases, each one with a different number of groups of 100 normal trajectories (ranging from 1 to 10) and outliers (from 1 to 10). For each test, ten different training/test sets were created, for a total of 2000 data sets...Note that outliers in the test sets are drawn from the same distribution used for outliers in the corresponding training sets, in order to ease the detection of anomalous training patterns being included in the normality class." [2].

The experiment was conducted in the following way: For each measure2, the $S L$ algorithm was applied over the test sets. Trajectories in clusters with size greater than 2, were classified as normal and the rest was classified as anomaly. The stopping criteria was selected by the following approach: A threshold $\epsilon$ was selected using the corresponding training set, and the $S L$ algorithm stopped before joining the pair of objects with the minimum dissimilarity greater than $\epsilon$. To compute $\epsilon$ in the corresponding training set: For each normal cluster $C_{i}$ : the mean of the dissimilarities between every pair of objects in $C_{i}$ was calculated,

${ }^{2}$ LCSS was tested with several choices of $\epsilon$ and $\delta . \epsilon=0.45$ and $\delta=2$ gave the best results. 
Table 2. Results using LCSS. For each entry (rows = anomalies; columns = groups of normal trajectories) ten runs were performed and the results averaged.

\begin{tabular}{|c|c|c|c|c|c|c|c|c|c|c|}
\hline \multirow{2}{*}{$\frac{\text { FALSE P }}{\text { Anomalies }}$} & & & & & & & & & & \\
\hline & \multicolumn{10}{|c|}{ Groups of normal trajectories } \\
\hline & 1 & 2 & 3 & 4 & 5 & 6 & 7 & 8 & 9 & 10 \\
\hline 1 & 0.00 & 0.00 & 0.00 & 0.00 & 0.00 & 0.00 & 0.00 & 0.00 & 0.00 & 0.00 \\
\hline$\overline{2}$ & 0.00 & 0.05 & 0.00 & 0.00 & 0.00 & 0.00 & 0.00 & 0.00 & 0.00 & 0.00 \\
\hline 3 & 0.00 & 0.03 & 0.00 & 0.00 & 0.00 & 0.00 & 0.00 & .00 & 0.00 & 0.00 \\
\hline 4 & 0.00 & 0.05 & 0.00 & 0.00 & 0.00 & 0.00 & 0.00 & 0.03 & 0.00 & 0.00 \\
\hline 5 & 0.02 & 0.02 & 0.02 & 0.04 & 0.00 & 0.00 & 0.00 & 0.00 & 0.02 & 0.00 \\
\hline 6 & 02 & 00 & & 0.02 & 0.00 & 0.00 & 0.00 & 0.00 & 0.00 & 0.00 \\
\hline$\overline{7}$ & & & & & & & 0.00 & 01 & 0.00 & 0.01 \\
\hline$\overline{8}$ & $\overline{0}$ & $\overline{0 .}$ & 0.00 & 0.00 & 0.00 & 0.00 & 0.00 & 0.00 & 0.00 & 0.00 \\
\hline$\overline{9}$ & 0.00 & & 0.02 & $\overline{0.00}$ & $\overline{0.00}$ & 0.00 & 0.02 & 0.00 & 0.00 & 0.00 \\
\hline 10 & 0.00 & 0.00 & 0.00 & 0.00 & 0.00 & 0.00 & 0.01 & 0.00 & 0.01 & 0.00 \\
\hline \multicolumn{11}{|c|}{ TRUE POSITIVES. The total average over 100 cases: $91.35 \%$} \\
\hline Anomalies & \multicolumn{10}{|c|}{ Groups of normal trajectories } \\
\hline 1 & 90.00 & & 83.33 & 97.50 & 98.00 & 83.33 & 97.14 & & & 95.0 \\
\hline$\overline{2}$ & 90.00 & 0.00 & 100.00 & 90.00 & $\overline{92.00}$ & $\overline{95.00}$ & $\overline{94.29}$ & 92.50 & 91.11 & $\overline{98.00}$ \\
\hline$\overline{3}$ & 100.00 & 0.00 & 96.67 & 95.00 & $\overline{94.00}$ & 91.67 & $\overline{90.00}$ & 95.00 & $\overline{93.33}$ & $\overline{95.00}$ \\
\hline$\overline{4}$ & 90.00 & & 96.67 & 92. & 92.00 & 98.33 & 98.57 & 93.75 & 93.33 & 90.00 \\
\hline 5 & 80.00 & 90.00 & 90.00 & 92.50 & 96.00 & 95.00 & 84.29 & 96.25 & 91.11 & 91.00 \\
\hline$\overline{6}$ & 90.00 & 100.00 & 100.00 & $\overline{85.00}$ & 86.00 & 88.33 & 92.86 & 88.75 & 95.56 & $\overline{96.00}$ \\
\hline 7 & 80.00 & & 86.67 & $\overline{95.00}$ & 92.00 & 85.00 & $\overline{92.86}$ & 93.75 & 94.44 & 89.01 \\
\hline 8 & 70.00 & 95.00 & 96.67 & 85.00 & 94.00 & 85.00 & 90.00 & 92.50 & 93.33 & 93.00 \\
\hline$\overline{9}$ & 90.00 & & 93.33 & 85.00 & 66.00 & $\overline{90.00}$ & 82.86 & 93.75 & 88.89 & 91.00 \\
\hline 10 & 80.00 & 90.00 & 90.00 & 90.00 & 86.00 & 86.67 & 87.14 & 93.75 & 92.22 & 00.00 \\
\hline
\end{tabular}

Table 3. Results using DTW. For each entry (rows = anomalies; columns = groups of normal trajectories) ten runs were performed and the results averaged.

\begin{tabular}{|c|c|c|c|c|c|c|c|c|c|c|}
\hline \multicolumn{11}{|c|}{ FALSE POSITIVES. The total average over 100 cases: $2.02 \%$} \\
\hline Anomalies & \multicolumn{10}{|c|}{ Groups of normal trajectories } \\
\hline & 1 & 2 & 3 & 4 & 5 & 6 & 7 & 8 & 9 & 10 \\
\hline 1 & 0.30 & 0.00 & 0.00 & 0.00 & 0.00 & 0.00 & 0.00 & 0.00 & 0.00 & 0.00 \\
\hline 2 & 0.35 & 1.45 & 1.35 & 1.10 & 1.40 & 5.35 & 1.85 & 0.95 & 1.25 & 2.85 \\
\hline 3 & 3.23 & 0.83 & 3.67 & 2.30 & 2.60 & 0.77 & 0.97 & 1.47 & 0.63 & 0.63 \\
\hline 4 & 4.13 & 1.73 & 2.68 & 3.15 & 2.63 & 1.58 & 1.88 & 2.25 & 4.43 & 1.60 \\
\hline$\overline{5}$ & 2.32 & 0.88 & 3.64 & 1.78 & 3.96 & 1.68 & 5.08 & 1.56 & 0.80 & 1.20 \\
\hline 6 & 2.57 & 1.62 & 2.20 & 2.08 & 2.32 & 2.03 & 2.80 & 2.47 & 0.60 & 3.23 \\
\hline 7 & 2.00 & 3.07 & 3.74 & 2.26 & 1.53 & 2.40 & 3.01 & 2.10 & 2.90 & 1.67 \\
\hline 8 & 2.05 & 2.19 & 2.46 & 2.98 & 3.14 & 3.75 & 2.11 & 2.19 & 3.06 & 1.58 \\
\hline$\overline{9}$ & 2.53 & 2.00 & 1.90 & 3.00 & 2.50 & 1.59 & 1.86 & 2.34 & 3.46 & 2.32 \\
\hline 10 & 3.87 & 1.48 & 2.03 & 1.76 & 2.96 & 1.13 & 3.10 & 2.54 & 1.34 & 2.18 \\
\hline \multicolumn{11}{|c|}{ TRUE POSITIVES. The total average over } \\
\hline Anomalies & \multicolumn{10}{|c|}{ Groups of normal trajectories } \\
\hline 1 & 90.00 & 100.00 & 100.00 & 100.00 & 100.00 & 100.00 & 100.00 & 98.75 & 100.00 & 100.00 \\
\hline 2 & 100.00 & 100.00 & 96.67 & 100.00 & 100.00 & 98.33 & 100.00 & 98.75 & 94.44 & 99.00 \\
\hline 3 & 100.00 & 100.00 & 96.67 & 95.00 & 96.00 & 98.33 & 98.57 & 98.75 & 97.78 & 99.00 \\
\hline$\overline{4}$ & 100.00 & 90.00 & 100.00 & 100.00 & 100.00 & 100.00 & 100.00 & 98.75 & 97.78 & 96.00 \\
\hline 5 & 80.00 & 100.00 & 96.67 & 92.50 & 98.00 & 96.67 & 95.71 & 98.75 & 96.67 & 96.00 \\
\hline 6 & 90.00 & 100.00 & 100.00 & 95.00 & 94.00 & 95.00 & 97.14 & 93.75 & 97.78 & 98.00 \\
\hline 7 & 100.00 & \begin{tabular}{|l|l}
95.00 \\
\end{tabular} & 100.00 & 97.50 & 96.00 & 90.00 & 95.71 & 97.50 & 96.67 & 97.00 \\
\hline 8 & 80.00 & 100.00 & 96.67 & 95.00 & 100.00 & 95.00 & 94.29 & 97.50 & 97.78 & 97.00 \\
\hline 9 & 100.00 & 100.00 & 96.67 & 100.00 & 86.00 & 98.33 & 88.57 & 95.00 & $\begin{array}{l}97.78 \\
\end{array}$ & 97.00 \\
\hline 10 & 100.00 & 90.00 & 93.33 & 95.00 & 94.00 & 90.00 & 92.86 & 95.00 & \begin{tabular}{|l|}
98.89 \\
\end{tabular} & 96.00 \\
\hline
\end{tabular}


Table 4. Results using the DT. For each entry (rows = anomalies; columns = groups of normal trajectories) ten runs were performed and the results averaged.

\begin{tabular}{|c|c|c|c|c|c|c|c|c|c|c|}
\hline \multirow{2}{*}{\multicolumn{11}{|c|}{ FALSE POSITIVES. The total average over $100 \mathrm{cec}$}} \\
\hline Anomalies & \multicolumn{5}{|c|}{ Groups of normal trajectories } & & & & & \\
\hline & 1 & 2 & 3 & 4 & 5 & 6 & 7 & 8 & 9 & 10 \\
\hline t & 0.30 & 0.00 & 0.00 & 0.00 & 0.00 & 0.00 & 0.00 & 0.00 & 0.10 & 0.00 \\
\hline 2 & 0.30 & 1.10 & 0.90 & 0.80 & 1.25 & 4.05 & 1.00 & 0.90 & 0.60 & 1.60 \\
\hline 3 & 1.93 & 0.40 & 1.67 & 1.13 & 1.43 & 0.37 & 0.70 & 0.87 & 0.27 & 0.53 \\
\hline 4 & 1.95 & 1.15 & 1.60 & 1.73 & 1.65 & 1.15 & 1.13 & 1.58 & 2.78 & 0.90 \\
\hline 5 & 1.56 & 0.70 & 2.18 & 1.24 & 2.74 & 1.04 & 2.64 & 0.82 & 0.58 & 0.86 \\
\hline 6 & 1.62 & 1.40 & 1.28 & 1.67 & 1.43 & 1.18 & 1.58 & 1.77 & 0.48 & 1.80 \\
\hline & 1.47 & 1.80 & 2.47 & 1.39 & 1.06 & 1.47 & 2.17 & 1.20 & 2.19 & 1.20 \\
\hline 8 & 1.48 & 1.30 & 1.64 & 1.70 & 1.66 & 2.33 & 1.30 & 1.20 & 2.03 & 0.84 \\
\hline 9 & 1.62 & 1.19 & 1.03 & 1.80 & 1.44 & 0.91 & 1.21 & 1.67 & 2.36 & 1.53 \\
\hline 10 & 2.38 & 1.07 & 1.28 & 1.16 & 1.86 & 0.77 & 2.04 & 1.72 & 0.92 & 1.59 \\
\hline \multicolumn{11}{|c|}{ TRUE POSITIVES. The total average over 100 cases: $96.65 \%$} \\
\hline Anomalies & & & & Troups & of norn & al traj & ctories & & & \\
\hline 1 & 90.00 & 100.00 & 100.00 & 100.00 & 100.00 & 100.00 & 100.00 & 98.75 & $\overline{100.00}$ & 100.00 \\
\hline$\overline{2}$ & 100.00 & 100.00 & 96.67 & 100.00 & 98.00 & 100.00 & 100.00 & 98.75 & 94.44 & 99.00 \\
\hline$\overline{3}$ & 100.00 & 100.00 & 96.67 & 95.00 & 96.00 & 98.33 & 98.57 & 98.75 & 97.78 & 99.00 \\
\hline & 100.00 & 90.00 & 100.00 & 100.00 & 100.00 & 100.00 & 100.00 & 98.75 & 97.78 & 96.00 \\
\hline 5 & 80.00 & 100.00 & 93.33 & 92.50 & 98.00 & \begin{tabular}{|l|l|}
96.67 \\
\end{tabular} & 92.86 & 98.75 & 96.67 & 96.00 \\
\hline 6 & 90.00 & 100.00 & 100.00 & 92.50 & 94.00 & 95.00 & 97.14 & 93.75 & 97.78 & 98.00 \\
\hline & 100.00 & 95.00 & 100.00 & \begin{tabular}{|l|}
97.50 \\
\end{tabular} & 96.00 & 90.00 & 95.71 & 97.50 & 96.67 & 96.00 \\
\hline 8 & 80.00 & 100.00 & 96.67 & 95.00 & 98.00 & 95.00 & 94.29 & 97.50 & 97.78 & 96.00 \\
\hline 9 & 100.00 & 100.00 & 96.67 & 100.00 & 88.00 & 98.33 & 88.57 & 96.25 & 97.78 & 97.00 \\
\hline 10 & 100.00 & 95.00 & 93.33 & 92.50 & 94.00 & 88.33 & 92.86 & 96.25 & \begin{tabular}{|l|l|}
97.78 \\
\end{tabular} & 97.00 \\
\hline
\end{tabular}

and the minimum of the calculated means was selected as $\epsilon$. Tables 1 through 8 show the results in terms of false positives ( $\mathrm{FP}=\%$ of normal trajectories misclassified as anomalies) and true positives ( $\mathrm{TP}=\%$ of abnormal trajectories correctly classified as anomalies).

The EU shows very good results detecting TP with an overall above $96 \%$ of effectiveness, but misclassifies many normal trajectories. This is very undesirable in real world applications, since many false alarms could lead human operators to a loss of confidence on the automatic system. On the contrary, LCSS has near zero FP, but stays more than $5 \%$ behind in TP detection. This behavior could be expected since LCSS is the only that does not penalizes the differences(section 2). DT and DTW behave very good in both aspects, but DTW have some particular cases where it misclassifies around $5 \%$ of normal trajectories. For the above mentioned reasons, the proposed dissimilarity measure shows better overall performance( $\mathrm{FP}, \mathrm{TP})$ than the others widely used measures.

\section{Conclusions}

In this paper, a new dissimilarity measure for trajectories was proposed and compared with three (dis)similarity measures widely used in the literature. The experiments for anomaly detection in video sequences via trajectory clustering (Single-Link), show that the proposed measure achieves the best results in overall performance. 


\section{References}

1. Vlachos, M., Hadjieleftheriou, M., Gunopulos, D., Keogh, E.: Indexing Multidimensional Time-Series. The VLDB Journal 15, 1-20 (2006)

2. Piciarelli, C., Micheloni, C., Foresti, G.L., Keogh, E.: Trajectory-Based Anomalous Event Detection. IEEE Transactions on Circuits And Systems For Video Technology 18, 1544-1554 (2008)

3. Chen, L., Tamer, M., Oria, V.: Robust and Fast Similarity Search for Moving Object Trajectories. In: 2005 ACM SIGMOD, pp. 491-502. ACM, New York (2005)

4. Zhang, Z., Huang, K., Tan, T.: Comparison of Similarity Measures for Trajectory Clustering in Outdoor Surveillance Scenes. In: 18th International Conference on Pattern Recognition, pp. 1135-1138. IEEE Computer Society, Los Alamitos (2006)

5. Morris, B.T., Trivedi, M.M.: A Survey of Vision-Based Trajectory Learning and Analysis for Surveillance. IEEE Transactions on Circuits and Systems For Video Technology 18, 1114-1127 (2008)

6. Xu, R., Wunsch II, D.C.: Clustering. IEEE Press, NJ (2009) 